This item was submitted to Loughborough's Research Repository by the author.

Items in Figshare are protected by copyright, with all rights reserved, unless otherwise indicated.

\title{
Phase coexistence and resistivity near the ferromagnetic transition of
} manganites

\section{PLEASE CITE THE PUBLISHED VERSION}

PUBLISHER

(c) Amerian Physical Society

LICENCE

CC BY-NC-ND 4.0

REPOSITORY RECORD

Alexandrov, A.S., A.M. Bratkovsky, and V.V. Kabanov. 2019. "Phase Coexistence and Resistivity Near the Ferromagnetic Transition of Manganites". figshare. https://hdl.handle.net/2134/1324. 


\title{
Phase Coexistence and Resistivity near the Ferromagnetic Transition of Manganites
}

\author{
A. S. Alexandrov, ${ }^{1}$ A. M. Bratkovsky, ${ }^{2}$ and V. V. Kabanov ${ }^{3}$ \\ ${ }^{1}$ Department of Physics, Loughborough University, Loughborough LE11 3TU, United Kingdom \\ ${ }^{2}$ Hewlett-Packard Laboratories, 1501 Page Mill Road, 1L, Palo Alto, California 94304, USA \\ ${ }^{3}$ Josef Stefan Institute, 1001 Ljubljana, Slovenia \\ (Received 31 January 2006; published 23 March 2006)
}

\begin{abstract}
Pairing of oxygen holes into heavy bipolarons in the paramagnetic phase and their magnetic pair breaking in the ferromagnetic phase (the so-called current-carrier density collapse) has accounted for the first-order ferromagnetic-phase transition, colossal magnetoresistance, isotope effect, and pseudogap in doped manganites. Here we propose an explanation of the phase coexistence and describe the magnetization and resistivity of manganites near the ferromagnetic transition in the framework of the currentcarrier density collapse. The present quantitative description of resistivity is obtained without any fitting parameters, by using the experimental resistivities far away from the transition and the experimental magnetization, and is essentially model-independent.
\end{abstract}

DOI: $10.1103 /$ PhysRevLett.96.117003

PACS numbers: 75.10. $-\mathrm{b}, 72.15 . J f, 75.47 .-\mathrm{m}$

Ferromagnetic oxides, in particular manganese perovskites, show very large magnetoresistance near the ferromagnetic transition. The effect observed in these materials was termed "colossal" magnetoresistance (CMR) to distinguish it from the giant magnetoresistance in metallic magnetic multilayers. The discovery raised expectations of a new generation of magnetic devices and is a focus of extensive research aimed at describing the effect. Significant progress has been made in understanding the properties of CMR manganites, but many questions remain. The ferromagnetic metal-insulator transition in manganites has long been thought to be a consequence of the so-called double-exchange mechanism (DEX), which results in a varying bandwidth of electrons in the $\mathrm{Mn}^{3+} d$ shell as a function of temperature [1]. More recently, it has been noticed [2] that the effective spin interaction cannot alone account for CMR within the double-exchange model. In fact, there is strong experimental evidence for exceptionally strong electron-phonon interactions in doped manganites from the giant isotope effect [3], the Arrhenius behavior of the drift and Hall mobilities [4] in the paramagnetic phase above the Curie temperature $T_{C}$, and other experiments. In view of this, Ref. [2] and some subsequent theoretical studies have combined DEX with the JahnTeller $e$-ph interaction with $d$ states, arriving at the conclusion that the low-temperature ferromagnetic phase is a spin-polarized metal, while the paramagnetic hightemperature phase is a polaronic insulator.

However, some low-temperature optical [5], electronenergy-loss (EELS) [6], photoemission [7], and thermoelectric [8] measurements showed that the ferromagnetic phase of manganites is not a conventional metal. In particular, broad incoherent spectral features and a pseudogap in the excitation spectrum were observed. EELS confirmed that manganites were, in fact, charge-transfer doped insulators having $p$ holes as current carriers rather than $d\left(\mathrm{Mn}^{3+}\right)$ electrons. Photoemission and x-ray absorption spectroscopies of $\mathrm{La}_{1-x} \mathrm{Sr}_{x} \mathrm{MnO}_{3}$ also showed that the itinerant holes doped into $\mathrm{LaMnO}_{3}$ have oxygen $p$ character. Further, CMR has been observed in the ferromagnetic pyrochlore manganite $\mathrm{Tl}_{2} \mathrm{Mn}_{2} \mathrm{O}_{7}$ [9], which has neither the mixed valence for DEX magnetic interaction nor the Jahn-Teller cations such as $\mathrm{Mn}^{3+}$.

These and other observations [10], in particular the fact that some samples of ferromagnetic manganites manifest an insulatinglike optical conductivity at all temperatures [11], clearly rule out DEX as the mechanism of CMR. The earlier of the above observations [3-6,9] led to a novel theory of ferromagnetic-paramagnetic phase transition and CMR, based on the so-called current-carrier density collapse (CCDC) [12], confirmed by later observations. In the CCDC model, the $p$ holes are bound into heavy bipolarons above the Curie temperature $T_{C}$ due to the Fröhlich electron-phonon interaction, which is written in the realspace representation as

$$
H_{e-\mathrm{ph}}=\sum_{\mathbf{n n}^{\prime} \sigma=\uparrow, \downarrow} f_{\mathbf{n}^{\prime}}(\mathbf{n}) c_{\mathbf{n} \sigma}^{\dagger} c_{\mathbf{n} \sigma} \xi_{\mathbf{n}^{\prime}}
$$

where $\xi_{\mathbf{n}^{\prime}}$ is the ion displacement operator, and the form of electron-phonon interaction is specified via the force function [13] $f_{\mathbf{n}^{\prime}}(\mathbf{n})$. The latter is defined as the force with which an electron in state $|\mathbf{n}\rangle$ interacts with the ion degree of freedom $\xi_{\mathbf{n}^{\prime}}$.

The resistivity peak and CMR are the result of the magnetic pair breaking below $T_{C}$ (Fig. 1) caused by the $p$ - $d$ spin-exchange interaction $J_{p d}$, described as

$$
H_{p d}=-(2 N)^{-1} \sum_{\mathbf{n}, \mathbf{m}} J_{p d} \hat{S}_{\mathbf{m}}^{z}\left(c_{\mathbf{n} \uparrow}^{\dagger} c_{\mathbf{n} \uparrow}-c_{\mathbf{n} \downarrow}^{\dagger} c_{\mathbf{n} \downarrow}\right) .
$$

Here $\hat{S}_{\mathbf{m}}^{z}$ is the $z$ component of $\mathrm{Mn}^{3+}$ spin on site $\mathbf{m}, c_{\mathbf{n} \uparrow}$ and $c_{\mathbf{n} \downarrow}$ annihilate a $p(\uparrow, \downarrow)$ hole on the oxygen site $\mathbf{n}$, with spin up and down, respectively, and $N$ is the total number of unit cells. 


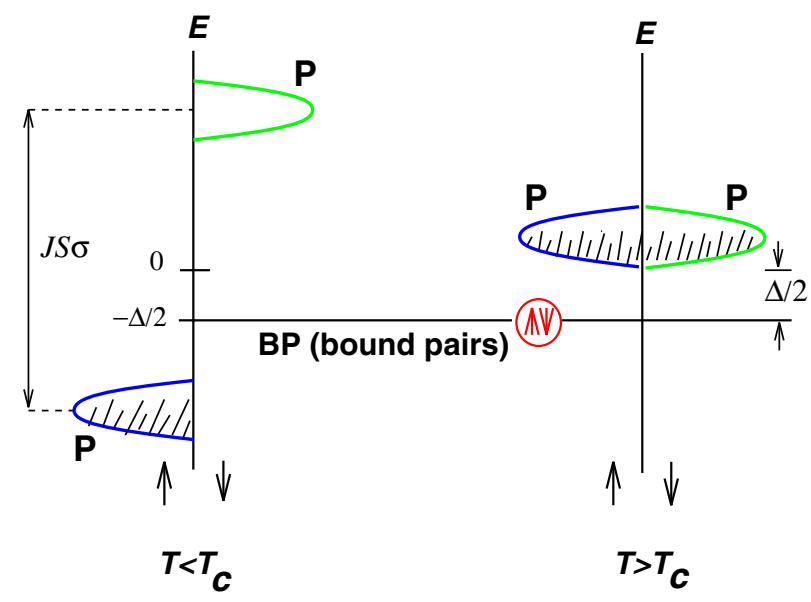

FIG. 1 (color online). Bipolaron model of CMR: Pairs (BP) are localized on impurity levels in the paramagnetic phase, where the only current carriers are single thermally excited polarons. If the exchange interaction $J S \sigma$ between $p$-hole polarons and ordered manganese spins exceeds the pair binding energy $\Delta$, the pairs break at $T<T_{C}$ because the spin-up polaron subband sinks abruptly below the bipolaron level. The ferromagnetic state is a polaronic conductor.

Different from cuprates, on-site or more extended intersite oxygen bipolarons are much heavier in manganites because the $e$-ph Fröhlich interaction [Eq. (1)] is stronger [14] and the band structure is less anisotropic. They are readily localized by disorder, so it is mainly thermally excited single polarons that conduct in the paramagnetic phase. Upon temperature lowering, single polarons polarize manganese spins at $T_{C}$ via the exchange interaction $J_{p d}$, and the spin polarization of manganese ions breaks the bipolaronic singlets, creating a spin-polarized polaronic conductor.

The CCDC model has explained CMR in the experimental range of external magnetic fields [12,15]. More recently, the theory has been further confirmed experimentally. In particular, the oxygen isotope effect has been observed in the low-temperature resistivity of $\mathrm{La}_{0.75} \mathrm{Ca}_{0.25} \mathrm{MnO}_{3}$ and $\mathrm{Nd}_{0.7} \mathrm{Sr}_{0.3} \mathrm{MnO}_{3}$ and explained by CCDC with polaronic carriers in the ferromagnetic phase [16]. The current-carrier density collapse has been directly observed using the Hall data in $\mathrm{La}_{0.67} \mathrm{Ca}_{0.33} \mathrm{MnO}_{3}$ and $\mathrm{La}_{0.67} \mathrm{Sr}_{0.33} \mathrm{MnO}_{3}$ [17], and the first-order phase transition at $T_{C}$, predicted by the theory [12], has been firmly established in the specific heat measurements [18]. Importantly, the character of the magnetic phase transition in $\mathrm{Tl}_{2} \mathrm{Mn}_{2} \mathrm{O}_{7}$ pyrochlores has also been determined to be of the first order [19] and attributed to the tendency of small polarons to phase separation at finite carrier density. Indeed, recent Monte Carlo simulations [20] of lattice polarons with anisotropic $e$-ph interactions and the realistic long-range Coulomb repulsion show diverse mesoscopic textures in the adiabatic limit, where spatially disordered pairs (i.e., bipolarons) dominate at finite doping.
On the other hand, resistivity and the magnetization of some $\mathrm{La}_{0.7} \mathrm{Ca}_{0.3} \mathrm{Mn}_{1-\delta} \mathrm{Ti}_{\delta} \mathrm{O}_{3}$ samples showed a more gradual (second-order-like) transition [21]. Also, the coexistence of ferromagnetic and paramagnetic phases near the Curie temperature observed in tunneling [22] and other experiments has not yet been addressed in the framework of CCDC. Here we show that diagonal disorder, which is inevitable with doping in those solid-state solutions, explains both the phase coexistence and the resistivity or magnetization behavior near the transition.

The mean-field equations [12] describing $p$-hole polaron atomic density $n$, polaron $m$, and manganese $\sigma$ reduced magnetizations and the chemical potential $\mu=$ $k_{B} T \ln y$ are readily generalized, taking into account a random distribution of the bipolaron binding energy $\delta=$ $\Delta /\left(2 J_{p d}\right)$ across the sample,

$$
\begin{gathered}
n_{i}=6 y \cosh \left(\sigma_{i} / t\right), \quad m_{i}=n_{i} \tanh \left(\sigma_{i} / t\right), \\
\sigma_{i}=B_{2}\left(m_{i} / 2 t\right), \quad y^{2}=\frac{x-n_{i}}{18} \exp \left(-2 \delta_{i} / t\right),
\end{gathered}
$$

where $t=k_{B} T / J_{p d}$ is the reduced temperature, $B_{S}$ is the Brillouin function, $x$ is the number of holes at zero temperature in $p$-orbital states, which are threefold degenerate. The subscript $i$ means different parts of the sample with different $\delta_{i}$ and, hence [12], with different Curie temperatures $T_{C i}$, owing to disorder.

While averaging these simple equations over a random distribution of $\delta_{i}$ is rather cumbersome, one can apply a simplified approach using the fact that the phase transition in a homogeneous system is of the first order in a wide range of $\delta$ [12]. Taking $\sigma_{i} \approx \Theta\left(T_{C i}-T\right)$ and $n_{i} \approx x \Theta\left(T_{C i}-T\right)+\sqrt{2 x} \exp \left[-\Delta /\left(2 k_{B} T\right)\right] \Theta\left(T-T_{C i}\right)$ and averaging both quantities with the Gaussian distribution of random $T_{C i} \mathrm{~s}$ around the experimental $T_{C}$, we obtain an averaged manganese magnetization

$$
\sigma(T)=\frac{1}{2} \operatorname{erfc}\left(\frac{T-T_{C}}{\Gamma}\right),
$$

where $\Delta$ is the average bipolaron binding energy, $\Theta(y)=1$ for $y>0$ and zero for $y<0$, and $\operatorname{erfc}(z)=\left(2 / \pi^{1 / 2}\right) \times$ $\int_{z}^{\infty} d y \exp \left(-y^{2}\right)$. The CCDC with disorder [Eq. (4)] fits nicely the experimental magnetizations [21] near the transition with physically reasonable $\Gamma$ of the order of $10 \mathrm{~K}$, depending on doping (Fig. 2). Hence, we believe that the random distribution of transition temperatures with the width $\Gamma$ across the sample caused by the randomness of the bipolaron binding energy is responsible for the phase coexistence near the transition as seen in the tunneling experiments [22]. We note that some drop of magnetization at low temperatures as seen in Fig. 2 might be caused by domain walls [23].

Resistivity of inhomogeneous two-phase systems has to be calculated numerically. Nevertheless, the comprehensive numerical simulations are consistent with a simple analytical expression for the resistivity of the binary 


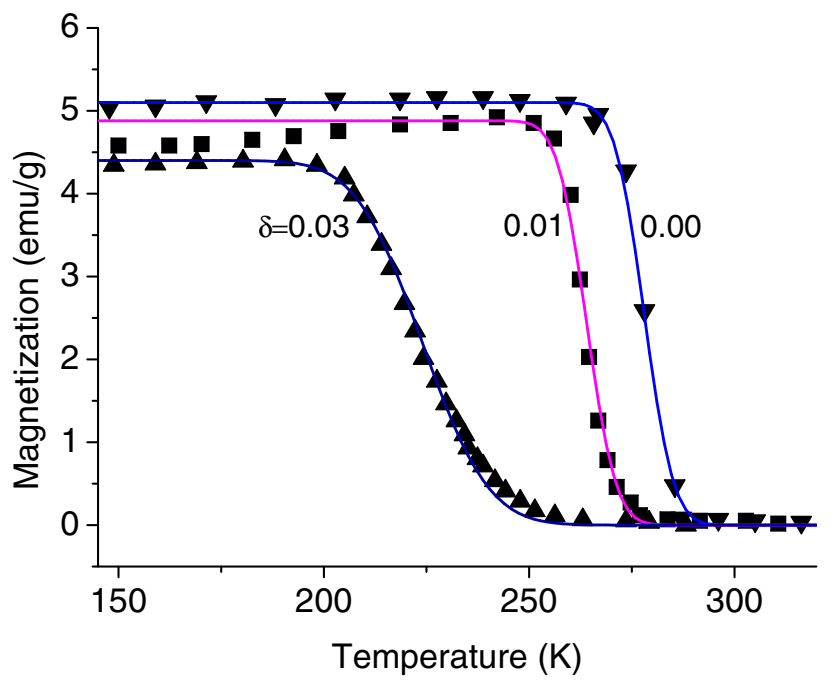

FIG. 2 (color online). Experimental magnetizations in $\mathrm{La}_{0.7} \mathrm{Ca}_{0.3} \mathrm{Mn}_{2-\delta} \mathrm{Ti}_{\delta} \mathrm{O}_{3}$ (symbols [21]) compared with Eq. (4) (lines) with $\Gamma=8 \mathrm{~K}, T_{C}=278 \mathrm{~K}$ for $\delta=0.00 ; \Gamma=$ $8 \mathrm{~K}, T_{C}=264 \mathrm{~K}$ for $\delta=0.01$; and $\Gamma=18 \mathrm{~K}, T_{C}=224 \mathrm{~K}$ for $\delta=0.03$.

mixture

$$
\rho=\rho_{1}^{1-\nu} \rho_{2}^{\nu},
$$

which is valid in a wide range of the ratios $\rho_{1} / \rho_{2}$ [24]. Here $\rho_{1,2}$ is the resistivity of each phase, respectively, and $\nu$ is the volume fraction of the second phase. The expression Eq. (5) is a homogeneous function of $\rho_{1,2}$ satisfying the duality relation. Scaling arguments [25] proved that the expression is exact near a percolation threshold in two dimensions. Numerical analysis [24] has shown that Eq. (5) describes the effective resistivity of 2D random systems even far away from the percolation threshold, if the resistivity ratio $\rho_{1} / \rho_{2}$ is not extremely large $(\$ 20)$. The same expression is also asymptotically correct for any 3D system, if $\left|1-\rho_{1} / \rho_{2}\right| \ll 1$, and for specific 3D lattice structures [26], if $\rho_{1} / \rho_{2} \gg 1$. Of course, there is no universal formula for any material with randomly distributed phases. Generally, one could write $f(\rho)=(1-\nu) f\left(\rho_{1}\right)+$ $\nu f\left(\rho_{2}\right)$, where $f(x)$ is a model function (for a comprehensive list of mixture formulas, see Refs. [27,28]). Equation (5) corresponds to the average of $\ln \rho$ in isotropic mixtures providing a qualitatively reasonable and numerically accurate description of the effective resistivity in many physically important cases (see below).

In the framework of CCDC, the resistivity of the paramagnetic phase is $\rho_{1}(T)=f(T) \exp \left(\Delta / 2 k_{B} T\right)$ and the resistivity of the ferromagnetic phase is $\rho_{2}(T)=\phi(T)$, where $f(T)$ and $\phi(T)$ are polynomial functions of temperature depending on the scattering mechanisms. Well below the transition, $\phi(T)$ can be parametrized as $\phi(T)=$ $\rho_{0}+a T^{2}$, and $f(T)=b T$ well above the transition, where the temperature-independent parameters $\rho_{0}, a, \Delta / 2$, and $b$ are taken directly from the experiment [21]. The micro- scopic origin of $\rho_{0}, a, b$, and alternative parametrization formulas have been discussed, e.g., in Refs. [21,29] and are not an issue here. The volume fraction $\nu$ of the ferromagnetic phase is simply the relative magnetization in our model $\nu=\sigma(T)$, also available from the experiment [21]. As a result, Eq. (5) provides the quantitative description of $\rho(T)$ in the transition region without any fitting parameters by using the experimental resistivities far away from the transition and the experimental magnetization [21], as shown in Fig. 3. On the other hand, fitting the ferromagnetic-phase resistivity with a magnetic scattering $\left(\rho_{4.5}^{m} T^{4.5}\right)$ leads to an unrealistic doping-dependent coefficient $\rho_{4.5}^{m}$ that is changing with doping by more than 5 orders of magnitude (see the table in Ref. [21]). Note that, if one were using an estimate $\rho \propto 1 / n$, where $n$ is the average single-polaron density, one would obtain

$$
\begin{aligned}
\rho^{-1}(T) \propto & \operatorname{erfc}\left(\frac{T-T_{C}}{\Gamma}\right) \\
& +(2 / x)^{1 / 2} e^{-\Delta / 2 k_{B} T} \operatorname{erfc}\left(\frac{T_{C}-T}{\Gamma}\right) .
\end{aligned}
$$

This expression can also fit the experimental curves but with a value of $T_{C}$, which turns out to be smaller than that in the magnetization [Eq. (4)] by several tens of degrees Kelvin [30]. The latter expression corresponds to a linear expansion of Eq. (5) in powers of $1-\rho_{1} / \rho_{2}$. It is easy to see why Eq. (5), when compared with Eq. (6), resolves the problem of different $T_{C} \mathrm{~s}$ in the magnetization and resistivity, thus providing a parameter-free description of experimental $\rho(T)$. If we take $\rho_{1} \gg \rho_{2}$, the resistivity at the magnetic transition (i.e., for $\nu=\sigma=1 / 2$ ), $\rho=$ $\rho_{2} \sqrt{\rho_{1} / \rho_{2}}$ calculated with Eq. (5) turns out larger than the resistivity $\rho \approx 2 \rho_{2}$ calculated with the perturbation expression Eq. (6). It is important that the present descrip-

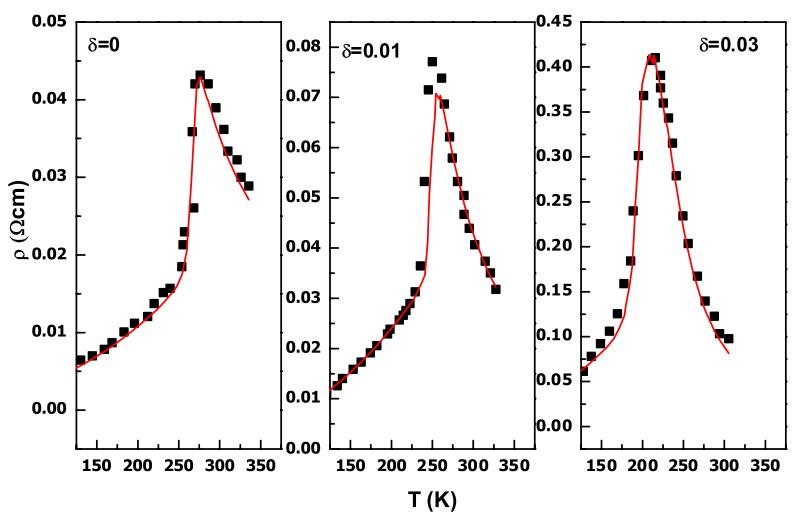

FIG. 3 (color online). The CCDC model [Eq. (5), lines] describes the experimental resistivity near the ferromagnetic transition in $\mathrm{La}_{0.7} \mathrm{Ca}_{0.3} \mathrm{Mn}_{2-\delta} \mathrm{Ti}_{\delta} \mathrm{O}_{3}$ (squares [21]), if the phase coexistence caused by disorder is taken into account. No fitting parameters are used in Eq. (5) but the experimental resistivity well below and well above the transition and the experimental magnetization. 
tion of resistivity does not depend on a particular model but on the assumption that the ferromagnetic transition is of the first order. The CCDC [12] has provided a basis for this assumption in terms of the microscopic model.

In summary, we have shown that the conventional DEX model, proposed half a century ago and generalized more recently to include the electron-phonon interaction, is in conflict with a number of recent experiments. Among these experiments are the site-selective spectroscopies, which have shown unambiguously that oxygen $p$ holes are the current carriers rather than $d$ electrons in ferromagnetic manganites. Also, some samples of ferromagnetic manganites manifest an insulatinglike optical conductivity at all temperatures, contradicting the DEX notion that their ferromagnetic phase is metallic. On the other hand, the pairing of oxygen holes into heavy bipolarons in the paramagnetic phase and their magnetic breakup in the ferromagnetic phase has explained the colossal magnetoresistance, the isotope effects, and the pseudogaps observed in doped manganites. It also explains the CMR in systems where DEX simply cannot exist, such as manganese pyrochlores [9]. The CCDC theory of CMR predicts the first-order phase transition and allows the present simple explanation of the coexistence of high- and lowresistive phases. It explains the temperature dependencies of the magnetization and the resistivity near the transition as the result of the unavoidable disorder and transport through the two-phase mixture in doped manganites.

This work was supported by EPSRC (U.K.) (Grants No. EP/D035589/1 and No. EP/C518365/1).

[1] C. Zener, Phys. Rev. 82, 403 (1951).

[2] A. J. Millis, P. B. Littlewood, and B. I. Shraiman, Phys. Rev. Lett. 74, 5144 (1995).

[3] G. M. Zhao, K. Conder, H. Keller, and K. A. Müller, Nature (London) 381, 676 (1996).

[4] M. Jaime, H. T. Hardner, M. B. Salamon, M. Rubinstein, P. Dorsey, and D. Emin, Phys. Rev. Lett. 78, 951 (1997).

[5] Y. Okimoto, T. Katsufuji, T. Ishikawa, T. Arima, and Y. Tokura, Phys. Rev. B 55, 4206 (1997); K. H. Kim, J.H. Jung, and T.W. Noh, Phys. Rev. Lett. 81, 1517 (1998); T. Ishikawa, T. Kimura, T. Katsufuji, and Y. Tokura, Phys. Rev. B 57, R8079 (1998).

[6] H. L. Ju, H.-C. Sohn, and K. M. Krishnan, Phys. Rev. Lett. 79, 3230 (1997).

[7] D. S. Dessau, T. Saitoh, C.-H. Park, Z.-X. Shen, Y. Moritomo, and Y. Tokura, Int. J. Mod. Phys. B 12, 3389 (1998); Y.D. Chuang, A. D. Gromko, D. S. Dessau, T. Kimura, and Y. Tokura, Science 292, 1509 (2001).
[8] J.-S. Zhou, J. B. Goodenough, A. Asamitsu, and Y. Tokura, Phys. Rev. Lett. 79, 3234 (1997).

[9] M. A. Subramanian, B. H. Toby, A.P. Ramirez, W. J. Marshall, A. W. Sleight, and G. H. Kwei, Science 273, 81 (1996).

[10] G. M. Zhao, Phys. Rev. B 62, 11639 (2000); G. M. Zhao, Y.S. Wang, D. J. Kang, W. Prellier, M. Rajeswari, H. Keller, T. Venkatesan, C. W. Chu, and R. L. Greene, Phys. Rev. B 62, R11949 (2000).

[11] A. Nucara, A. Perucchi, P. Calvani, T. Aselage, and D. Emin, Phys. Rev. B 68, 174432 (2003).

[12] A. S. Alexandrov and A. M. Bratkovsky, Phys. Rev. Lett. 82, 141 (1999); J. Phys. Condens. Matter 11, 1989 (1999).

[13] A.S. Alexandrov, Theory of Superconductivity: From Weak to Strong Coupling (Institute of Physics, Bristol, 2003).

[14] A. S. Alexandrov and A. M. Bratkovsky, Phys. Rev. Lett. 84, 2043 (2000); J. Phys. Condens. Matter 11, L531 (1999).

[15] L. M. Wang, H.C. Yang, and H. E. Horng, Phys. Rev. B 64, 224423 (2001).

[16] A.S. Alexandrov, G. M. Zhao, H. Keller, B. Lorenz, Y.S. Wang, and C. W. Chu, Phys. Rev. B 64, 140404(R) (2001).

[17] W. Westerburg, F. Martin, P. J. M. van Bentum, J. A. A. J. Perenboom, and G. Jakob, Eur. Phys. J. B 14, 509 (2000).

[18] J.E. Gordon, C. Marcenat, J. P. Franck, I. Isaac, G. W. Zhang, R. Lortz, C. Meingast, F. Bouquet, R. A. Fisher, and N.E. Phillips, Phys. Rev. B 65, 024441 (2002).

[19] P. Velasco, J. Mira, F. Guinea, J. Rivas, M. J. MartinezLope, J. A. Alonso, and J. L. Martinez, Phys. Rev. B 66, 104412 (2002).

[20] T. Mertelj, V. V. Kabanov, and D. Mihailovic, Phys. Rev. Lett. 94, 147003 (2005).

[21] X. M. Liu, H. Zhu, and Y.H. Zhang, Phys. Rev. B 65, 024412 (2002).

[22] M. Uehara, S. Mori, C. H. Chen, and S. W. Cheong, Nature (London) 399, 560 (1999).

[23] See, for example, A. Senchuk, H.P. Kunkel, R. M. Roshko, C. Viddal, Li Wei, G. Williams, and X.Z. Zhou, Eur. Phys. J. B 37, 285 (2004).

[24] V. V. Kabanov, K. Zagar, and D. Mihailovic, Zh. Eksp. Teor. Fiz. 127, 809 (2005) [JETP 100, 715 (2005)].

[25] A. L. Efros and B. I. Shklovskii, Phys. Status Solidi B 76, 475 (1976).

[26] J. B. Keller, J. Math. Phys. (N.Y.) 28, 2516 (1987).

[27] G. W. Milton, The Theory of Composites (Cambridge University Press, Cambridge, England, 2002).

[28] F. G. Shin, W. L. Tsui, and Y. Y. Yeung, J. Mater. Sci. Lett. 8, 1383 (1989).

[29] Guo-meng Zhao, V. Smolyaninova, W. Prellier, and H. Keller, Phys. Rev. Lett. 84, 6086 (2000).

[30] A. S. Alexandrov, cond-mat/0506706. 